\title{
18
}

\section{The intelligent discussion supporting system over the computer network}

\author{
T. Okamoto and A. Inaba
}

The Graduate School of Information Systems, University of ElectroCommunications, 1-5-1 Chofugaoka, Chofu, Tokyo 182 JAPAN

Phone: (81)-424832161_Fax: (81)-424815934

email: okamoto@ai.is.uec.ac.jp

\begin{abstract}
The purpose of this paper is to propose a technical and psychological framework of intelligent computer supported collaborative work/learning environment which we call "iDCLE" (intelligent distributed collaborative learning/working environment). To realize iDCLE, we constructed a real-time sharing communication system for collaborative work/learning on computer networks. We describe the mechanism for iDCLE in this paper, and discuss the mechanism of supporting discussion. Using our model, the discussion support system in iDCLE can identify participants' states of discussion. The system can also diagnose the process of discussion for the purpose of making progress. When advice is needed after the diagnosis, the system gives the appropriate feedback to the participants.
\end{abstract}

\section{Keywords}

Distance learning, artificial intelligence, collaborative Learning

\section{INTRODUCTION}

Recently, networking technology like the Internet has developed rapidly and we can communicate and discuss with others in a distributed environment. Under such a network environment, we can communicate with people in foreign countries and share various kinds of educational knowledge like new teaching methods, educational materials, curriculum contents, and evaluation methods. The educational environment is changing from a closed system to a global and open system.

With those considerations, we propose an "iDCLE" (intelligent Distributed Collaborative Learning/working Environment) where people can exchange knowledge and solve problems through effective group thinking. Many researchers on educational technology attempt to extend the field of study from a stand-alone learning environment to the group learning environment, where multiple agents interact with one another (Baker, 1994, 1995; Blandford, 1994). The concept of CSCL (Computer Supported Collaborative Learning) has attracted the 
interest of many researchers who wish to study a mechanism of dynamic group learning and develop more flexible communication technology. This concept is based on the rationales of knowledge constructivism and social learning. In the case of group learning under the networking environment, though it is necessary to provide multi-modal means of communication, we suppose that verbal communication is especially important (Lave and Wenger, 1991; Salomon, 1993). It is quite important for the educational system to be designed bearing in mind the cognitive interaction among the participants in order to improve their communication skills.

New interactive technologies based on remote computing as networking and multimedia have helped to provide the interactive communication previously lacking in distance education. Fortunately, computer-mediated multimedia communication systems are starting to be exploited by the various kinds of organizations/institutes as alternative delivery environments independent of time and distance (Okamoto, 1994).

In the study of CSCL, some researchers aim to construct a system which is equipped with a transparent and seamless environment for supporting effective collaborative work/learning. Other researchers aim to construct a system which can monitor and coordinate appropriately the process of group work/learning using artificial intelligence technology. We used the latter approach in order to enhance group productivity and the self-monitoring ability of each participant.

In this study, we constructed the iDCLE framework in which the participants attain the educational goal through interaction with one another (Okamoto, Inaba and Hasaba, 1995; Inaba, Hasaba and Okamoto, 1996). In collaborative learning, it is necessary to communicate with others. Thus, how to support the discussion is one of the most important issues in research on collaborative learning. We embed the expert system which plays the role of coordinator to support discussion based on the model of transition on dialogue states in iDCLE.

\section{RESEARCH OBJECTIVES}

Our study has three research objectives:

- to construct a dialogue model for discussion on a computer network;

- to develop an intelligent discussion supporting system which can identify the state of discussion and help the discussion by the model described above;

- to clarify appropriate strategies for guiding the discussion based on collaborative knowledge.

\section{THE iDCLE SYSTEM}

In this section, we describe the fundamental configuration of the iDCLE system. The system is constructed on UNIX workstations connected by TCP/IP. The core of its graphical user interface (GUI) is constructed on the X11 window systems.

In iDCLE, each participant can freely choose either of two workspaces or both. One is the Personal Work Space, which is a closed private area and cannot be accessed by other participants. This enables the participants to explore their individual information and to 
learn/work something individually as a performance support system. The participant can use several tools for problem solving in this space, e.g. a word processor, a drawing tool, and so on. Another work space is the Collaborative Work Space shared by all participants. This space is used to exchange opinions and to solve problems collaboratively. This space has two places, which are called the Communication Channel and the Clear Board.

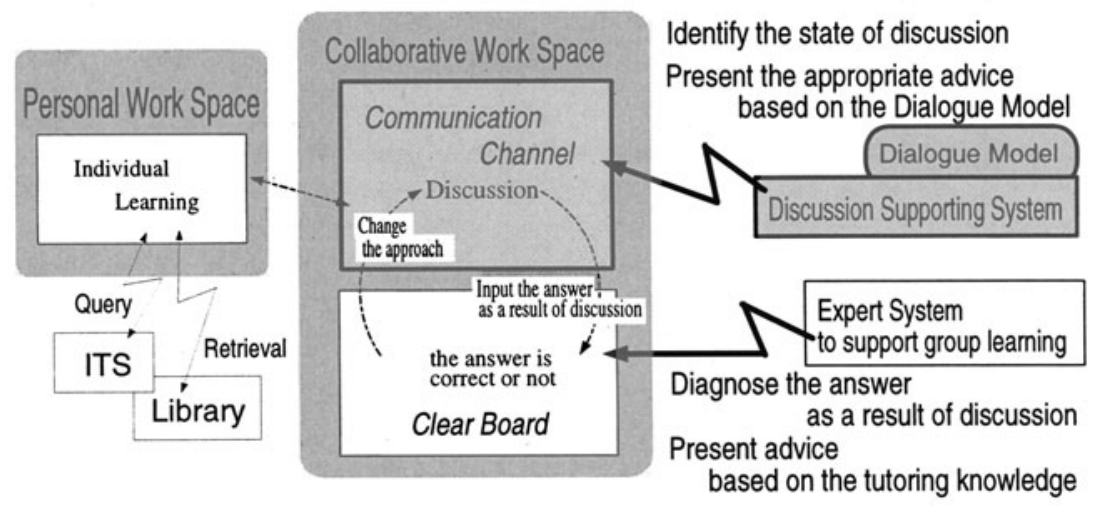

Figure 1 The Learning Environment.

Figure 1 shows the learning environment in iDCLE. The participants discuss a given task through the Communication Channel. The discussion support system including the computer coordinator can identify the state of discussion and give the appropriate advice by tracing the dialogue model. That is, the participants present some sentences through the Communication Channel. The system can extract intention-information from utterance sentences by processing natural language for context understanding of conversation. Then the sequence of extracted intention is stored on working memory in a discussion support system. The discussion support system diagnoses a state of discussion and identifies a participant's role in a discussion process based on the sequence of intention-information on the working memory. The system generates appropriate advice through this Communication Channel. As a result of the discussion, the participants input the answer to the Clear Board. The Clear Board is a space which enables participants to share an application software such as an expert system. The shared expert system diagnoses whether their answers are correct or not, then gives some advice to coordinate group learning appropriately if it is necessary. When the answer is not correct, the participants may discuss again and change the answer.

\section{SUPPORTING DISCUSSION}

As mentioned above, the discussion on the Communication Channel is supported by the computer coordinator in iDCLE. This mechanism is described in this section.

We have constructed a discussion support system as a prototype (Okamoto, Inaba and Hasaba, 1995). The system can diagnose four states where the system should intervene:

- the divergent state of discussion, i.e. when each participant gives his/her idea, and does not say anything related to utterances of the other participants; 
- the impasse state of discussion, i.e. when a participant explains something about his/her opinion, and the other participants disagree with the explanation;

- the state in which a participant gives little utterance for a while;

- the state in which a question is not answered.

We conducted some experiments to evaluate the system's functions of coordinating the flow of discussion. As a result, it was suggested that the system should have the function to identify each participant's role in the discussion process. Therefore, we have improved the model of transition on dialogue states and the rule bases on the discussion supporting system which we call the Coordinator system.

\subsection{Model of Dialogue}

We propose a model of transition on dialogue states by analyzing data of protocols taken from some cognitive experiments. We classify the various kinds of information existing in participants' utterance into two categories. One is concerned with the semantics of the utterance. The information included in this category is related to both the domain knowledge and the context of discussion. Another kind of information is concerned with the participant's intention in making the utterance. Considering a method of supporting discussion, it seems inevitable that the system utilizes the intention-information included in the participants' utterance to coordinate the discussion flow. We contrived the sophisticated mechanism by which the intention-information can be separated from the contents of a given problem to propose the general purposed framework of iDCLE.

The supporting functions of our system are:

- to make users participate actively in the discussion;

- to facilitate group discussion smoothly in the desired direction (such as finding the adequate solution for a problem).

To realize the functions mentioned above, the system should have the function to identify the state of discussion. We classify the participants' intention into ten categories as shown in Table 1 .

Table 1 The categories of utterance's intention

\begin{tabular}{|c|c|c|c|}
\hline Function & $\begin{array}{l}\text { The function } \\
\text { of starting a topic }\end{array}$ & $\begin{array}{l}\text { The function of expressing } \\
\text { a participant's position on } \\
\text { other's opinion }\end{array}$ & Other function \\
\hline Category & $\begin{array}{l}\text { Proposition } 1^{*} \\
\text { Proposition } 2 \dagger \\
\text { Confirmation } \\
\text { Question }\end{array}$ & $\begin{array}{l}\text { Agreement } \\
\text { Disagreement }\end{array}$ & $\begin{array}{l}\text { Explanation } \\
\text { Answer } \\
\text { Supplement } \\
\text { Others }\end{array}$ \\
\hline
\end{tabular}

\footnotetext{
* Proposition about a way to develop the discussion

$\dagger$ Proposition of next topic for the problem solving
}

We propose the model of transition on dialogue states bearing in mind the ten categories described in Table 1. We regard a discussion as the sequential structure of some topics. So, we have only to explore the structure of the transition on dialogue states in a topic. The model of transition on dialogue states consists of four modes: the Guide mode, the Development 
mode, the Confirmation mode and the Query mode. The structure of each of the four modes is shown in Figure 2.

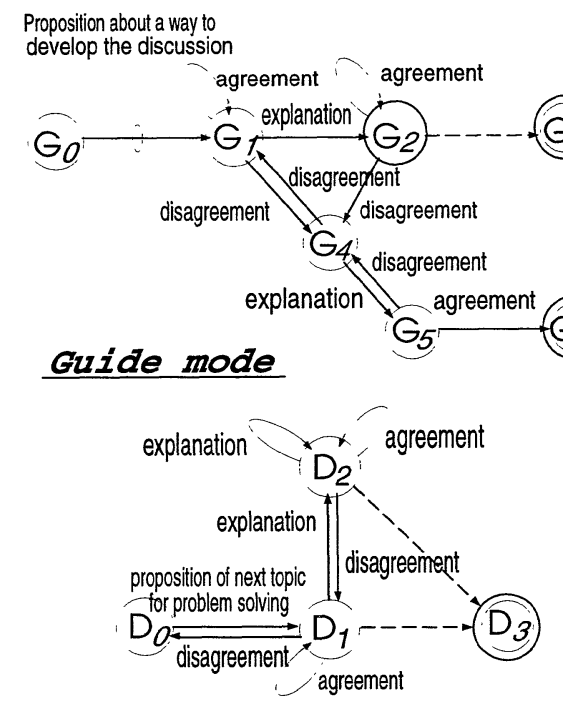

Development mode

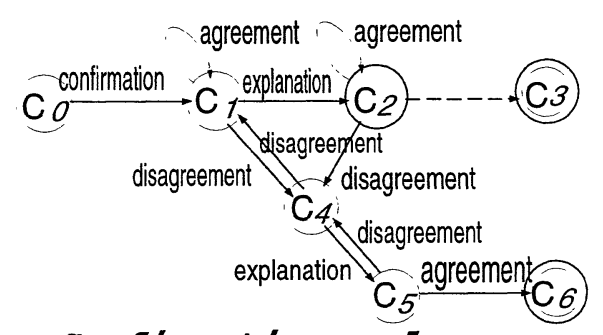

confirmation mode

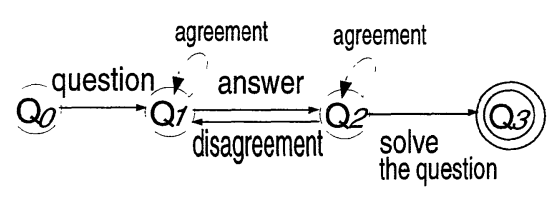

Query mode

Figure 2 The model of transition on dialogue state.

\section{Guide mode}

The Guide mode means dialogue transitions related to making progress in the discussion process. The model on Guide mode works when a participant gives the intention-information of the proposition for developing discussion. The discussion moves from one state to another according to intention. For instance, when the current state is G1 and somebody gives an explanation, then the state moves from G1 to G2. As another instance, when the current state is $\mathrm{G} 2$ and somebody disagrees with the current proposition, then the state moves from $\mathrm{G} 2$ to G4. When a state transition on this mode exists and a new intention-information of a proposition is given (i.e. the dialogue focus moves to a new topic), the system checks the current state. If the current state is G1 or G2, then the state transition reaches final state G3. On the other hand, if the current state is G4 or G5, then the state transition reaches final state G6. When the current proposition is accepted by the participants, the state transition reaches G3. If the participants do not accept the current proposition, the state transition reaches G6.

\section{Development mode}

The Development mode means dialogue transitions related to finding an appropriate way for problem solving. The model on the Development mode works when a participant gives the intention-information of a proposition of next topic for problem solving. If the state is D1 or D2, and almost all participants agree with the proposition, then the state transition reaches the final state. When a state transition exists and a new proposition is given, a new state transition 
gets working and the old one is suspended and labeled "WAIT". This labeled state of WAIT means the state which all of the participants do not agree with the current proposition, therefore the system suspended this one.

\section{Confirmation mode}

The Confirmation mode means dialogue transitions related to specifying an idea offered by a participant. The model on the Confirmation mode works when a participant gives the intentioninformation for the confirmation. Similar to Guide mode, when the current confirmation is judged to be correct by the other participants, the state transition reaches $\mathrm{C} 3$. When the participants judge the confirmation to be incorrect, the state transition reaches C6.

\section{Query mode}

The Query mode means dialogue transitions in which a participant asks a question, and the other participants try to answer the question. The model on the Query mode works whenever the participant asks. When the state is Q2 and the participant, who asked the question, solves it, the state transition reaches the final state.

\subsection{Expert System as Coordinator}

Each participant can interactively communicate the intention of utterance by selecting the appropriate button in the intention-menu which has the ten categories mentioned in section 4.1. The sequence of inputted intention is stored on working memory in the Coordinator system which is the expert system to coordinate the flow of the discussion.

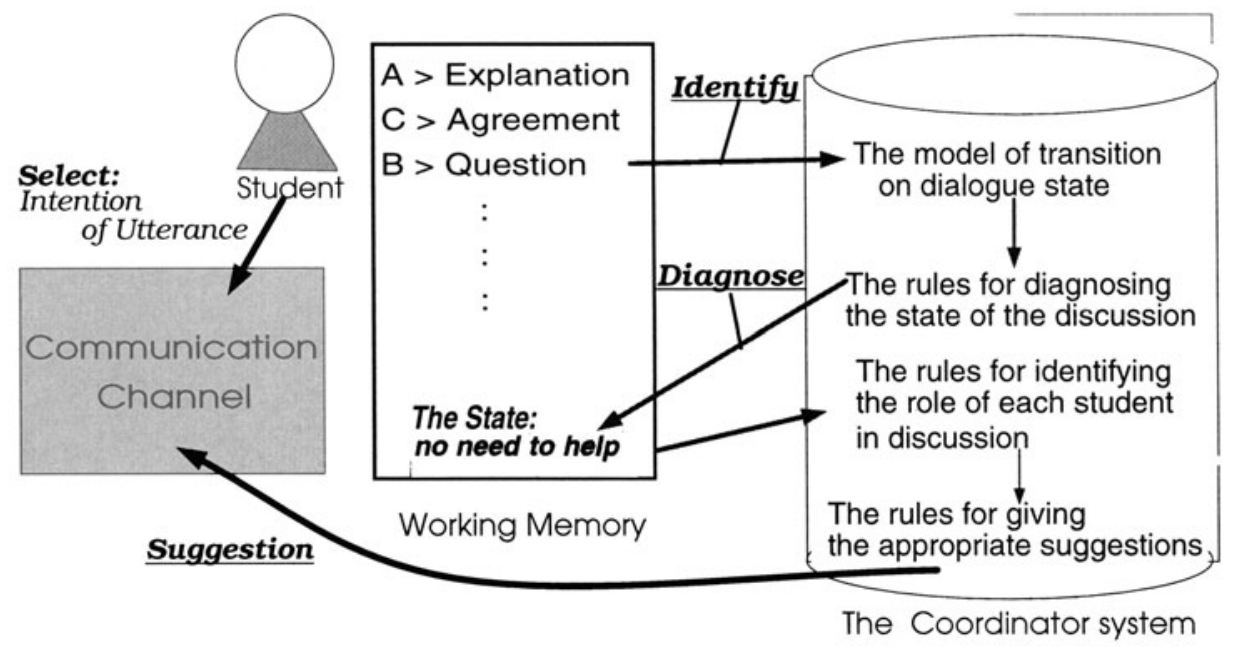

Figure 3 The Coordinator system.

We show the framework of the Coordinator system in Figure 3. The Coordinator system has four kinds of rules as follows. 
Rule set-A: The rules for tracing the model of transition on dialogue states.

Rule set-B: The rules for diagnosing the state of discussion.

Rule set-C: The rules for identifying the roles of each participant in discussion.

Rule set-D: The rules for giving the appropriate advice.

These rules were extracted from the results of cognitive experiments on group learning/discussion. The system can identify the state of discussion by referring rule set-A in the production system, and diagnose when the system should intervene by referring rule set-B. The system identifies the roles of each participant in discussion(e.g. a leader in the group) by referring rule set-C. Finally the system gives appropriate advice to the participant who plays a specific role in the discussion process by referring rule set-D.

When the system decides to give the participants some advice, it tries to classify all participants' roles into the following categories:

- the participant who leads the discussion process;

- the participant who has the initiative in the current topic;

- the participant who understands the contents of discussion well;

- the participant who does not understand the contents of discussion;

- the participant who often asks;

- the participant who gives little utterance;

- the participant who gives propositions which the other participants do not accept;

- the participant who does not belong to the above categories.

Then, the system gives the appropriate advice to each participant with the role corresponding to each category standing for the state of discussion.

\section{CONCLUSION}

In this paper, we proposed a model of transition on dialogue states and a framework of the system which coordinates/supports group learning intelligently using the production system with four layers of rule sets. We contrived the model of transition on dialogue states which utilizes the intention-information included in participants' utterance. We have developed an intelligent discussion supporting system for iDCLE based on this framework. Our intelligent discussion supporting system, called the Coordinator system, would be able to contribute to understand and coordinate the discussion process by realizing the following functions;

- The system can recognize participants' discussion process;

- The system can diagnose some discussion states in which the system should intervene;

- The system can identify each participant's role during discussion process;

- The system can give appropriate advice for each participant's role on self-involvement in discussion.

As a task for further research, it is important to clarify more appropriate strategies for guiding discussion flow based on collaborative knowledge. 


\section{REFERENCES}

Baker, M. (1994) A model for negotiation in teaching-learning dialogues. Journal of Artificial Intelligence in Education, 5(2), 199-254.

Baker, M. (1995) Negotiation in collaborative problem-solving dialogues, in Dialogue and Instruction (ed. R.J.Beun, et al.), Springer-Verlag, Berlin.

Blandford, A.E. (1994) Teaching through collaborative problem solving. Journal of Artificial Intelligence in Education, 5(1), 51-84.

Inaba, A., Hasaba, Y. and Okamoto, T. (1996) An intelligent supporting of discussion for the distributed cooperative learning environment. The Transactions of IEICE, J79-A(2), 207215.

Lave, J. and Wenger, E. (1991) Situated learning. Cambridge Univ. Press, Cambridge.

Okamoto, T. (1994) Global communication literacy, in Proceedings of Symposium, JET/Japan Academy of Cooperation Foundation, 20-26.

Okamoto, T., Inaba, A. and Hasaba, Y. (1995) The intelligent learning support system on the distributed cooperative environment, in Proceedings of Artificial Intelligence in Education 95, 588 .

Salomon, G. (Ed.) (1993) Distributed cognitions. Cambridge Univ. Press, Cambridge.

\section{BIOGRAPHIES}

Toshio Okamoto graduated in 1971 from Kyoto University of Education and completed coursework in graduate school in 1975 at Tokyo Gakugei University. He obtained Dr. of Engineering from Tokyo Institute of Technology. Presently, he is a Professor in the Information Systems Laboratory, University of Electro-Communications. He is engaged in research on AI models for intelligent CAI systems. He translated Artificial Intelligence and Intelligent CAI Systems. He is a member on the Board of CAI Society, and Japanese Society of Educational Technology. He also is a member of several committees including that of Educational Engineering, Artificial Intelligence \& Knowledge Engineering in IEICE, and a member of Society of Artificial Intelligence.

Akiko Inaba graduated in 1991 and completed coursework in graduate school in 1993 at Ibaraki University in Japan. She obtained a Master's degree of education. Presently she is a doctoral student at the Graduate school of Information Systems, University of ElectroCommunications. She is engaged in research on Computer Supported Collaborative Learning. 